\title{
The more we use, the more we lose
}

\author{
Ali Alkan ${ }^{1} \cdot$ Dılşa Mızrak ${ }^{1}$ Güngör Utkan ${ }^{1}$
}

Received: 7 March 2015/Accepted: 11 March 2015/Published online: 19 March 2015

(C) Springer Science+Business Media New York 2015

To the Editor,

We have read the study by Meattini et al. [1] with great interest. Nausea and vomiting (NV) are the most common chemotherapy-induced toxicities and constitute an important part of oncology practice. Aprepitant, in addition to 5HT3 inhibitors, made chemotherapy regimens more tolerable. The efficacy in highly emetogenic regimens has been confirmed in moderately emetogenic regimens, and more widely usage has provided more NM control. Fluorouracil, epirubicin, and cyclophosphamide (FEC) combination is important in breast cancer management, and grades 3-4 NM ranges between 9.9 and $20.5 \%$ in clinical trials [2,3]. The addition of aprepitant to steroid and setron combination provided better $\mathrm{NV}$ results in the study by Meattini et al. However, multidrug therapies are like a double-edged sword, and drug interactions should be cautiously checked. The addition of aprepitant to FEC regimen can result in other toxicities and even change the expected therapy results. Aprepitant inhibits CYP450 3A4, and coadministration of aprepitant with drugs metabolized with CYP450 3A4 can result in increased levels of the target drug. Pharmacokinetics of cyclophosphamide can be effected with aprepitant, and this can cause unexpected toxicities while preventing NV. Another issue in this multidrug regimen is the increased incidence of QT prolongation in both palonosetron and epirubicin. This cardiac side effect is another toxicity that must be followed cautiously.

In oncology practice, our aim was to treat patients with minimum toxicity and maximum efficacy. While doing this, drug interactions must be checked, and expected toxicities and interactions should be cautiously followed.

Conflict of interest The authors declare that they have no conflict of interest.

\section{References}

1. Meattini I, Francolini G, Scotti V, De Luca Cardillo C, Cappelli S, Meacci F, et al. Aprepitant as prophylaxis of chemotherapyinduced nausea and vomiting in anthracyclines and cyclophosphamide-based regimen for adjuvant breast cancer. Med Oncol. 2015;32(3):535.

2. Roche H, Fumoleau P, Spielmann M, Canon JL, Delozier T, Serin $\mathrm{D}$, et al. Sequential adjuvant epirubicin-based and docetaxel chemotherapy for node-positive breast cancer patients: the FNCLCC PACS 01 Trial. J Clin Oncol. 2006;24(36):5664-71.

3. Martin M, Rodriguez-Lescure A, Ruiz A, Alba E, Calvo L, RuizBorrego M, et al. Randomized phase 3 trial of fluorouracil, epirubicin, and cyclophosphamide alone or followed by Paclitaxel for early breast cancer. J Natl Cancer Inst. 2008;100(11):805-14.

Ali Alkan

alkanali@yahoo.com

1 Ankara University School of Medicine, Medical Oncology, Ankara, Turkey 